\title{
Ophiopogon japonicus inhibits radiation-induced pulmonary inflammation in mice
}

\author{
Qi-Wei Yao ${ }^{1,2}$, Xiao-Ying Wang ${ }^{3}$, Jian-Cheng Li $^{1,2}$, Jun Zhang ${ }^{4}$ \\ ${ }^{1}$ Department of Radiation Oncology, Fujian Cancer Hospital \& Fujian Medical University Cancer Hospital, Fuzhou 350014, China; ${ }^{2}$ Provincial \\ Clinical College, Fujian Medical University, Fuzhou 350122, China; ${ }^{3}$ Department of Radiation Oncology, The First Hospital of Putian, Teaching \\ Hospital, Fujian Medical University, Putian 351100, China; ${ }^{4}$ Division of Hematology, Oncology and Blood \& Marrow Transplantation, Department \\ of Internal Medicine, Holden Comprehensive Cancer Center, University of Iowa Carver College of Medicine, Iowa City, IA, USA \\ Contributions: (I) Conception and design: JC Li; (II) Administrative support: JC Li; (III) Provision of study materials or patients: J Zhang; (IV) \\ Collection and assembly of data: XY Wang; (V) Data analysis and interpretation: QW Yao; (VI) Manuscript writing: All authors; (VII) Final approval \\ of manuscript: All authors. \\ Correspondence to: Jian-Cheng Li. Department of Radiation Oncology, Fujian Cancer Hospital \& Fujian Medical University Cancer Hospital, Fuzhou \\ 350014, China. Email: Jianchengli6@126.com; Jun Zhang. Division of Hematology, Oncology and Blood \& Marrow Transplantation, Department \\ of Internal Medicine, Holden Comprehensive Cancer Center, University of Iowa Carver College of Medicine, Iowa City, IA 52242, USA. \\ Email: jun-zhang-1@uiowa.edu.
}

Background: Radiation-induced lung injury, including the acute pulmonary inflammation and chronic pulmonary fibrosis remains the major complication of thoracic radiotherapy.

Methods: In this study, we assessed the effects of Ophiopogon japonicus (O. japonicas) in inhibiting the radiation-induced pulmonary inflammation through an acute lung injury mouse model using C57BL/6 mice that received 18 Gy irradiation to the thoracic region. Starting at 4 days before radiation, mice were treated with O. japonicus or dexamethasone combined with cephalexin or vehicle daily for 14 days.

Results: Exposure to radiation resulted in pulmonary inflammation in mice, but treatment with O. japonicus or dexamethasone-cephalexin could both significantly reduce radiation-induced pulmonary inflammation through inhibition of IL-6, TNF- $\alpha$, TGF- $\beta 1$, hydroxyproline, MDA, MMP-2 and TIMP-2 in plasma or lung tissue. In addition, through analyzing tissue damage, cytokines and inflammation-related protein at 12 weeks after irradiation, we found that the protective effect of $\mathrm{O}$. japonicus was more enduring than dexamethasone-cephalexin.

Conclusions: As radiation-induced lung injury is a major obstacle in thoracic radiotherapies and seriously affect the quality of patients' life. Application of O. japonicus may be a novel strategy to manage radiationinduced pulmonary inflammation.

Keywords: Ophiopogon japonicus (O. japonicas); radiotherapy; pulmonary inflammation

Submitted Sep 04, 2018. Accepted for publication Oct 10, 2019.

doi: 10.21037/atm.2019.11.01

View this article at: http://dx.doi.org/10.21037/atm.2019.11.01

\section{Introduction}

Thoracic radiotherapy is widely used for the treatment of lung cancer. However, it can also cause different levels of radiation-induced lung injury in lung tissue adjacent to tumor. Radiation dose delivered to the tumor was therefore partly limited by the radiation-induced lung injury including the acute pulmonary inflammation and chronic pulmonary fibrosis, which seriously affect the quality of patients' life, and in worst scenario, could even lead to death $(1,2)$. At present, glucocorticoids, antibiotics, bronchodilators and immunosuppressive agents are commonly used to treat acute radiation-induced lung injury. However, these strategies have suboptimal effect with side effects that may adversely affect the quality of patients' life $(3,4)$. 
The mechanism of radiation-induced pneumonitis has not been fully understood until recently. It is generally believed that radiation-induced pneumonitis is a multifactorial process that involves target cell damage, reactive oxygen species activation, and cytokines generation. Therefore, inflammation-related protein and inflammatory cytokine, such as TGF- $\beta 1$, TNF- $\alpha$ and IL- 6 released by infiltrated inflammatory cells, might be effective targets whose inhibition could theoretically prevent radiation pneumonitis (5-7).

Ophiopogon japonicus (O. japonicas) is an evergreen perennial plant in the Liliaceae family, which has been used as a traditional Chinese medicine to treat various inflammatory diseases for thousands of years (8). It has been reported that $\mathrm{O}$. japonicas has certain roles in inhibiting tumor growth and reducing toxicity of chemotherapy $(9,10)$. This work aims to evaluate the protective effect of O. japonicas on radiation-induced pulmonary inflammation using an acute lung injury mouse model.

\section{Methods}

\section{Experimental animals and reagents}

Male C57BL/6 mice (age, 8 weeks; weight, 20-25 g) were purchased from Shanghai SLAC Experimental Animal Center and maintained in a specific pathogen-free grade animal room. O. japonicas was purchased from Quanzhou Southeast Chinese Medicinal Materials Planting Co., Ltd., Fujian, China. Dexamethasone was purchased from Xianju Pharmaceutical Co., Ltd., Zhejiang, China. Cephalexin was purchased from Yatai Pharmaceutical Co., Ltd., Zhejiang, China.

\section{Experimental groups}

Sixty C57BL/6 mice were divided into four groups: (I) control group: administration by gavage of $0.4 \mathrm{~mL}$ saline. (II) Radiation group: administration by gavage of $0.4 \mathrm{~mL}$ saline with 18 Gy irradiation to the thoracic region of $6 \mathrm{MV}$ $\mathrm{X}$-ray. (III) O. japonicas group: administration by gavage of $1.85 \mathrm{mg} / \mathrm{mL}$ O. japonicas $0.4 \mathrm{~mL}$ with $18 \mathrm{~Gy}$ irradiation to the thoracic region of $6 \mathrm{MV} \mathrm{X}$-ray. (IV) Dexamethasonecephalexin (combination treatment) group: administration by gavage of $0.4 \mathrm{~mL}$ saline contains $0.06 \mathrm{mg} / \mathrm{mL}$ dexamethasone and $12.33 \mathrm{mg} / \mathrm{mL}$ cephalexin with $18 \mathrm{~Gy}$ irradiation to the thoracic region of 6 MV X-ray. Starting at 4 days before radiation all treatments were given once a day for 14 days.

\section{Preparation of lung tissues for histology}

Left-lung tissues of irradiated mice were fixed in $10 \%$ formalin and then embedded in paraffin. For histological study, sections were stained with hematoxylin and eosin (HE) and immunohistochemical (IHC) stains.

\section{Preparation of lung tissues for detection of bydroxyproline, $M D A, M M P-2$ and TIMP-2}

The concentrations of hydroxyproline and MDA in the lung tissues were measured using the activity assay kit (Nanjing Jiancheng Bioengineering Institute, Nanjing, China). All of the procedures were conducted according to the instructions of the manufacturer. For detection of MMP-2 and TIMP-2, tissue sections were incubated with an antiMMP-2 or anti-TIMP-2 primary antibody (1:100 dilution, Santa Cruz, Dallas, TX, USA) at $4{ }^{\circ} \mathrm{C}$ overnight. Slides were then incubated with secondary antibodies labeled with HRP (Abcam, Cambridge, UK) and were developed using 3, 3-diaminobenzidine tetrachloride (DAB; DAKO, Carpinteria, CA, USA). Integrated optical density (IOD) was measured by image analysis software (Image-Pro Plus; Media Cybernetics, MD, USA), and the positive area was compared with the total area for semi-quantitative analysis.

\section{Preparation of plasma for detection of IL-6, TNF- $\alpha$ and TGF- $\beta 1$}

Mice blood samples were collected and then centrifuged at $1,500 \mathrm{rpm}$ for $5 \mathrm{~min}$. Plasma were then collected and prepared for testing IL- $6, \mathrm{TNF}-\alpha$ and TGF- $\beta 1$ concentrations by enzyme-linked immunosorbent assay (ELISA).

\section{Statistical analysis}

All the measurement data are expressed as the means \pm standard error of mean (SEM). The $t$-test or oneway analysis of variance (ANOVA) was applied to analyze the differences between groups, using data statistics software SPSS 17.0. $\mathrm{P}<0.05$ represents a significant difference. 


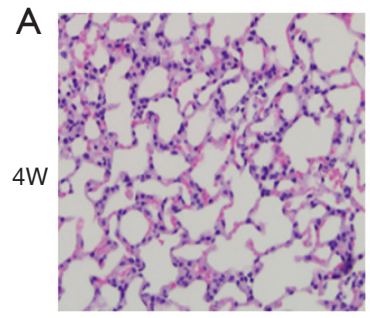

Control

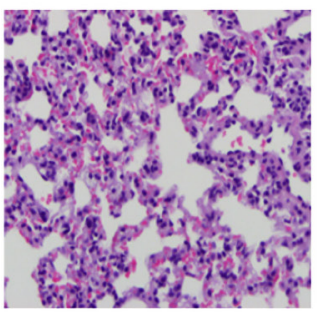

Radiation

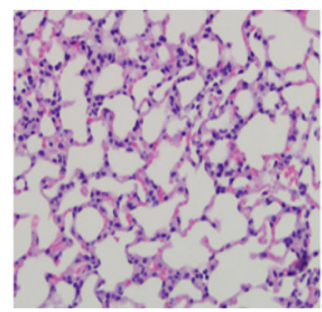

Combination treatment

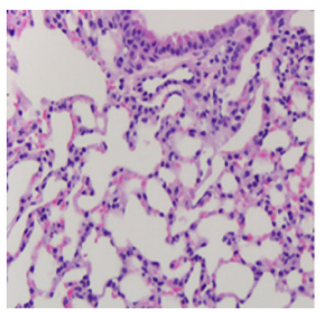

O. japonicas

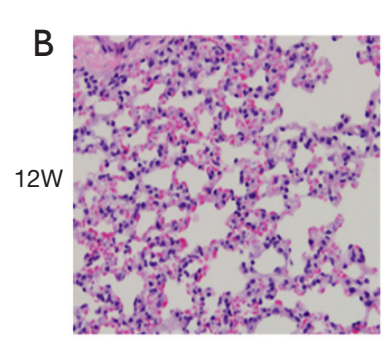

Control

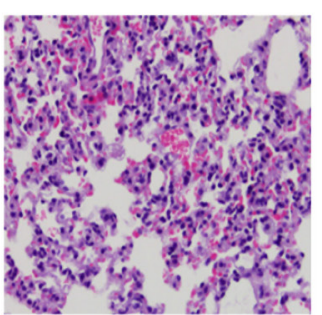

Radiation

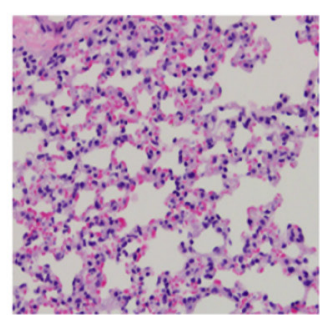

Combination treatment

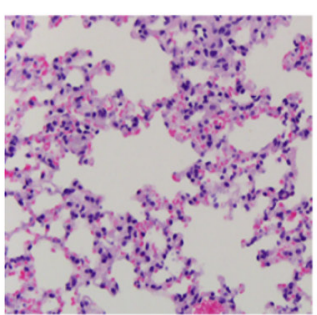

O. japonicas

Figure 1 Effects of O. japonicas on radiation-induced histological changes in lung tissue at (A) 4 weeks and (B) 12 weeks after irradiation. The representative histological changes were shown using HE staining $(\times 100)$. 4W, 4 weeks; $12 \mathrm{~W}$, 12 weeks post radiation; O. japonicas, Ophiopogon japonicus; HE, hematoxylin and eosin.

\section{Results}

\section{Effects of O. japonicus on radiation-induced histological changes in lung tissue}

To study the effect of $\mathrm{O}$. japonicas on radiation-induced pneumonitis, we first compared the histological changes of lung tissue after radiation exposure with or without treatment of $\mathrm{O}$. japonicas or dexamethasone-cephalexin (combination treatment group) (11). C57BL/6 mice showed acute pulmonary inflammation at 4 weeks after irradiation that manifested as accumulation of neutrophils in alveolar spaces, as well as capillary congestion (Figure $1 A$ ). At 12 weeks after irradiation, mice with radiation alone were found having altered pulmonary interstitium, reduced alveolar space, increased cellulose exudation, as well as infiltration of macrophages and fibroblasts into the alveolar space (Figure 1B). In contrast, treatment with O. japonicas or dexamethasone-cephalexin both reduced tissue damage, including lesser degrees of pulmonary interstitial congestion, edema and intra-alveolar inflammatory cell infiltration at 4 weeks after irradiation (Figure 1A). Notably, compared to dexamethasone combined with cephalexin, treatment with $\mathrm{O}$. japonicas showed a significant durable reduction of tissue damage at 12 weeks after irradiation (Figure 1B).

\section{Effects of O. japonicus on inflammation-related protein expression in plasma}

To understand the underlying mechanism, we compared the inflammation-related protein expression in mice plasma after radiation with or without treatment of $\mathrm{O}$. japonicas or dexamethasone-cephalexin. Expression of inflammationrelated cytokines (IL- 6 , TNF- $\alpha$ and TGF- $\beta 1$ ) was significantly increased in irradiated mice plasma. Treatment with $\mathrm{O}$. japonicas or dexamethasone-cephalexin depressed the increasing expression of IL- 6 , TNF- $\alpha$ and TGF- $\beta 1$ at 4 weeks after irradiation. Importantly, compared to the combination treatment, treatment with O. japonicas resulted in a much enduring inhibition in the up-regulation of cytokines at 12 weeks after irradiation (Figure 2).

\section{Effects of O. japonicus on inflammation-related protein expression in lung tissue}

To study the effect of $\mathrm{O}$. japonicas on radiation-induced inflammation-related protein expression in lung tissue, we compared the inflammation-related protein expression of lung tissue after radiation with or without treatment of O. japonicas or dexamethasone-cephalexin. Expression of inflammation-related protein (hydroxyproline, MDA, MMP-2 and TIMP-2) was significantly increased in 

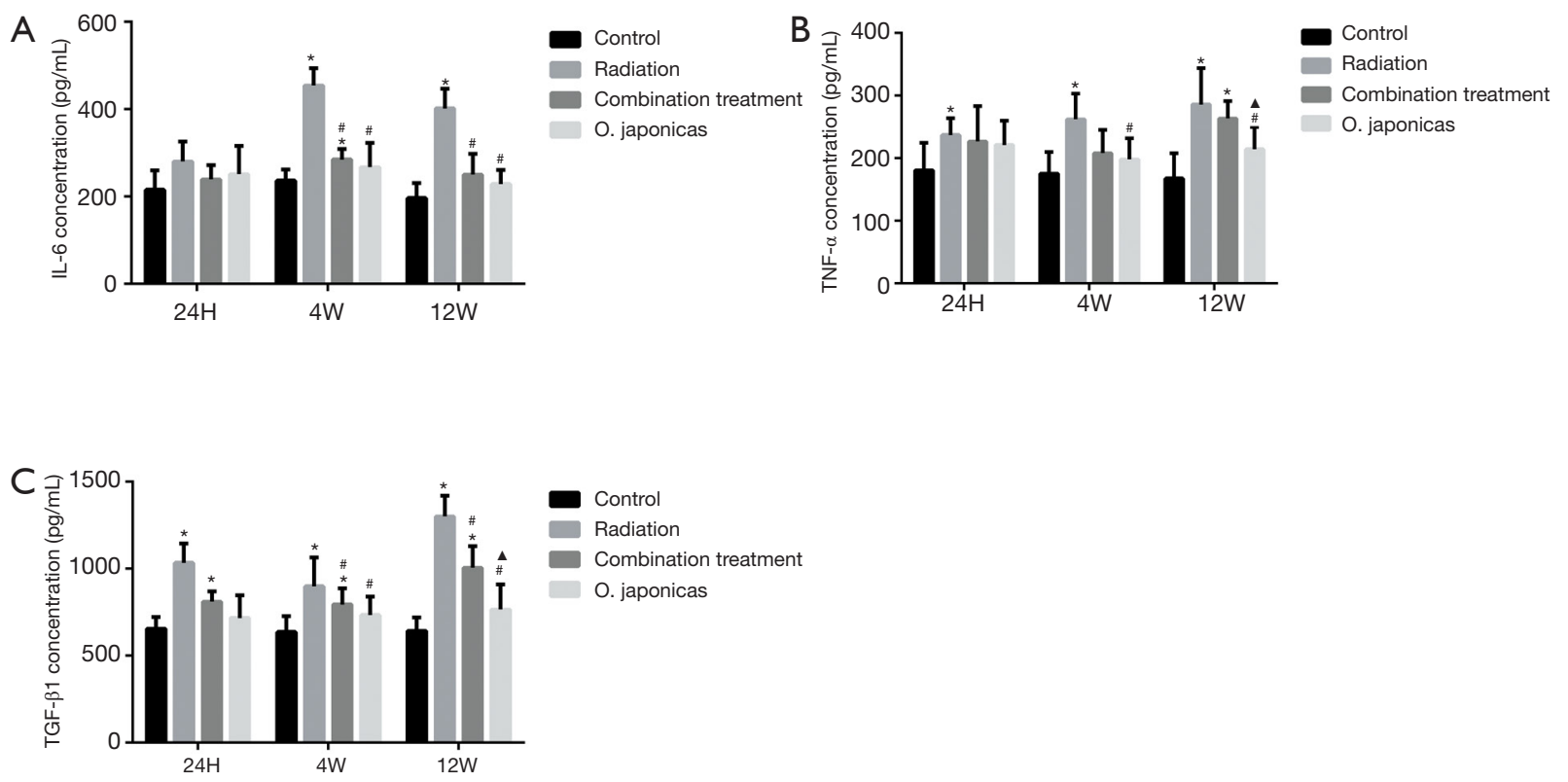

Figure 2 Effects of O. japonicas on inflammation-related protein expression in plasma. Expression of (A) IL-6, (B) TNF- $\alpha$ and (C) TGF- $\beta 1$ in plasma was measured. The presented data were obtained from three replicates and expressed as the mean $\pm \mathrm{SEM}$. *, $\mathrm{P}<0.05$, compared to the control group; *, $\mathrm{P}<0.05$, compared to the radiation group; $\boldsymbol{\Delta}, \mathrm{P}<0.05$, compared to the combination treatment group. O. japonicas, Ophiopogon japonicus; SEM, standard error of mean; 24H, 24 hours; 4W, 4 weeks; 12W, 12 weeks post radiation.

irradiated mice lung tissue. Treatment with O. japonicas or dexamethasone-cephalexin could both inhibit the upregulation of hydroxyproline, MDA, MMP-2 and TIMP2 at 4 weeks after irradiation (Figure 3). Notably, compared to combination treatment, $\mathrm{O}$. japonicas achieved a more sustained inhibition of hydroxyproline and MDA expression when detected at 12 weeks after irradiation (Figure $3 A, B$ ). Its effect on MMP-2 and TMP-2 was similar to the combination treatment.

\section{Discussion}

Radiation-induced lung injury is a major obstacle in thoracic radiotherapy. Current approach to prevent and treat such injury has been focused on certain key factors, such as the recombinant human keratinocyte growth factor (rHuKGF), which can promote the proliferation and differentiation of type II epithelial cell of the lung (12); or free radical scavengers, which can increase the free radical content of lung tissue after radiotherapy; as well as agents which are capable of inhibiting TGF- $\beta 1$ and its relevant signal transduction proteins (2). However, it is now clear that radiation-induced lung injury is not a simple pathologic process driven by a single factor but rather a cascade effect of a network of cells and cytokines in the lung tissue.

In this study, we constructed a model of acute radiation pneumonia in mice. We found that the lung tissue of irradiated mice showed acute pulmonary inflammation changes at 4 weeks after irradiation, which manifested as accumulation of neutrophils in alveolar spaces and capillary congestion. At 12 weeks after irradiation, histopathological examination showed altered pulmonary interstitium, reduced alveolar space, increased cellulose exudation, as well as migration of macrophages and fibroblasts into the alveolar space. We also found that the concentration of plasma IL-6, TNF- $\alpha$, TGF- $\beta 1$ and the expression of hydroxyproline, MDA, MMP-2, TIMP-2 protein in lung tissue were all increased.

Cytokine-mediated multi-cell interactions are involved in the development of radiation-induced pulmonary inflammation. Studies have shown that radiation promotes lung alveolar epithelial cells, fibroblasts, alveolar macrophages and vascular endothelial cells to synthesize and secrete large amounts of cytokines, including those involved in local injury and inflammatory response (such as TNF- $\alpha$, IL-1, IL-6, IL-8, etc.) and factors involved in tissue repair and organ fibrosis (such as TGF- $\beta 1$ ) (13). Rübe et al. have shown that bronchial epithelial cells could 
A

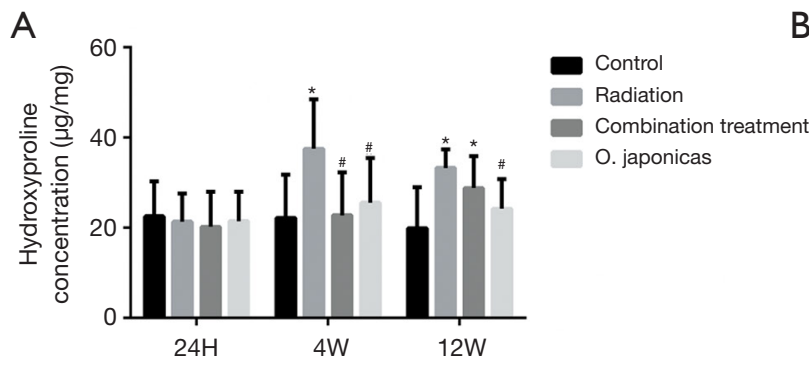

$\mathrm{B}$ 음

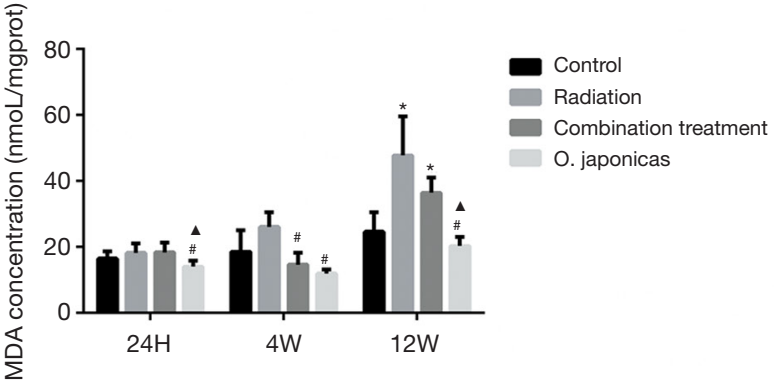

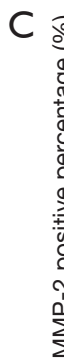
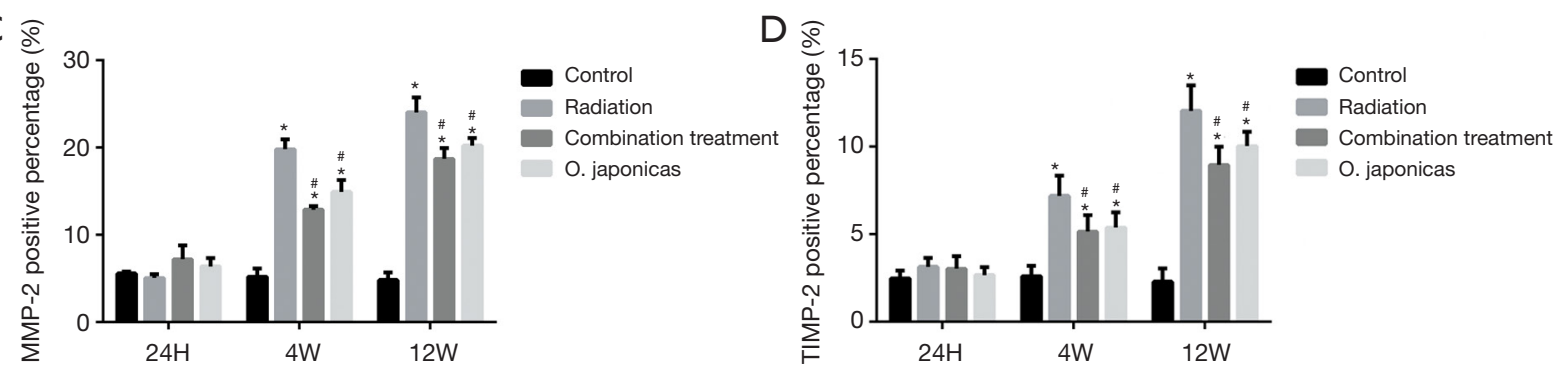

Figure 3 Effects of O. japonicas on inflammation-related protein expression in lung tissue. Expression of (A) hydroxyproline, (B) MDA, (C) MMP-2 and (D) TIMP-2 in plasma was measured. The presented data were obtained from three replicates and expressed as the mean \pm SEM. *, $\mathrm{P}<0.05$, compared to the control group; ${ }^{\#}, \mathrm{P}<0.05$, compared to the radiation group; $\mathbf{\Delta}, \mathrm{P}<0.05$, compared to the combination treatment group. O. japonicas, Ophiopogon japonicus; SEM, standard error of mean; 24H, 24 hours; 4W, 4 weeks; 12W, 12 weeks post radiation.

release proinflammatory cytokines, TNF- $\alpha$, IL-1, and IL6 , after 1 hour of pulmonary irradiation, which initiated and maintained intratracheal inflammation in radiation-induced lung injury (14). Anscher et al. found that TGF- $\beta 1$ and its mRNA expression were up-regulated in the pulmonary fibrosis model, indicating that TGF- $\beta 1$ has the function of promoting collagen production and deposition (15). They also measured changes in plasma TGF- $\beta 1$ level in patients with lung cancer before, during, and after radiotherapy, and found that plasma TGF- $\beta 1$ level was increased at the end of radiotherapy in patients with radiation-induced pneumonia (16). Our study found that the levels of IL6 , TNF- $\alpha$ and TGF- $\beta 1$ in plasma of the mice in radiation group were higher than those in the control group, indicating that our model recapitulated some key features of radiation-induced pulmonary inflammation. Treatment with $\mathrm{O}$. japonicas or dexamethasone-cephalexin inhibited the up-regulation of IL- 6 , TNF- $\alpha$ and TGF- $\beta 1$ at 4 weeks after irradiation. Interestingly, we also found that compared to the combination treatment, $\mathrm{O}$. japonicas could achieve a more sustained suppression of those inflammatory cytokines at 12 weeks after irradiation, which suggested that the protective effect of $\mathrm{O}$. japonicus could be more enduring than dexamethasone in combination with cephalexin.

Hydroxyproline is an important component of collagen which correlates to the severity of the lung fibrosis (17), and MDA is an important lipid peroxide that reflects the damage caused by oxygen free radical (18). Our results showed that the content of hydroxyproline and MDA in lung tissue in the radiation group was significantly higher than that in the control group. Treatment with O. japonicas or dexamethasone -cephalexin suppressed the up-regulation of hydroxyproline and MDA in lung tissue at 4 weeks after irradiation. In addition, $\mathrm{O}$. japonicas was found having more sustained suppression in hydroxyproline and MDA when compared to the combination treatment at 12 weeks after irradiation.

Some studies have shown the levels of MMPs are elevated in the BALF from COPD patients (19,20), high expression of both MMP and TIMP have been found in sputum from chronic bronchitis which are correlated with impaired lung function (21). Our results showed that the expression of 
MMP-2 and TIMP-2 in lung tissue in the radiation group was significantly higher than that in the control group. Treatment with $\mathrm{O}$. japonicas or dexamethasone-cephalexin both inhibited the up-regulation of MMP-2 and TIMP-2 in lung tissue at 4 and 12 weeks after irradiation.

In conclusion, treatment with $\mathrm{O}$. japonicas or dexamethasone-cephalexin could both significantly attenuate the lung inflammatory response to radiation through reducing the expression of plasma proinflammatory cytokines IL-6, TNF- $\alpha$ and fibrosis promoting cytokine TGF- $\beta 1$. Treatment with O. japonicas or dexamethasonecephalexin could also inhibit the up-regulation of hydroxyproline and MDA, as well as MMP-2 and TIMP-2 in lung tissue. What's more, the protective effect of $\mathrm{O}$. japonicas was found more enduring than dexamethasone combined with cephalexin. These results suggested that $\mathrm{O}$. japonicas may be a novel therapeutic strategy for radiation-induced pulmonary inflammation.

\section{Acknowledgments}

None.

\section{Footnote}

Conflicts of Interest: The authors have no conflicts of interest to declare.

Ethical Statement: The authors are accountable for all aspects of the work in ensuring that questions related to the accuracy or integrity of any part of the work are appropriately investigated and resolved. This study was supported by the Fuzhou General Hospital (No. IACUC-2014-0010).

\section{References}

1. Palma DA, Senan S, Tsujino K, et al. Predicting radiation pneumonitis after chemoradiation therapy for lung cancer: an international individual patient data meta-analysis. Int $\mathrm{J}$ Radiat Oncol Biol Phys 2013;85:444-50.

2. Zhang XJ, Sun JG, Sun J, et al. Prediction of radiation pneumonitis in lung cancer patients: a systematic review. J Cancer Res Clin Oncol 2012;138:2103-16.

3. Graves PR, Siddiqui F, Anscher MS, et al. Radiation pulmonary toxicity: from mechanisms to management. Semin Radiat Oncol 2010;20:201-7.

4. Deng B, Deng C, Cheng Z. Chinese herbal extractions for relieving radiation induced lung injury: a systematic review and meta-analysis. Evid Based Complement Alternat Med 2017;2017:2141645.

5. Ding NH, Li JJ, Sun LQ. Molecular mechanisms and treatment of radiation-induced lung fibrosis. Curr Drug Targets 2013;14:1347-56.

6. Wang Y, Wang X, Wang X, et al. Effect of transforming growth factor- $\beta 1869 \mathrm{C} / \mathrm{T}$ polymorphism and radiation pneumonitis. Int J Clin Exp Pathol 2015;8:2835-9.

7. Kainthola A, Haritwal T, Tiwari M, et al. Immunological aspect of radiation-induced pneumonitis, current treatment strategies, and future prospects. Front Immunol 2017;8:506.

8. Chen MH, Chen XJ, Wang M, et al. Ophiopogon japonicus--a phytochemical, ethnomedicinal and pharmacological review. J Ethnopharmacol 2016;181:193-213.

9. Chen M, Guo Y, Zhao R, et al. Ophiopogonin B induces apoptosis, mitotic catastrophe and autophagy in A549 cells. Int J Oncol 2016;49:316-24.

10. Chen J, Yuan J, Zhou L, et al. Regulation of different components from Ophiopogon japonicus on autophagy in human lung adenocarcinoma A549Cells through PI3K/ Akt/mTOR signaling pathway. Biomed Pharmacother 2017;87:118-26.

11. Liao ZX, Travis EL, Tucker SL. Damage and morbidity from pneumonitis after irradiation of partial volumes of mouse lung. Int J Radiat Oncol Biol Phys 1995;32:1359-70.

12. Chen L, Brizel DM, Rabbani ZN, et al. The protective effect of recombinant human keratinocyte growth factor on radiation-induced pulmonary toxicity in rats. Int J Radiat Oncol Biol Phys 2004;60:1520-9.

13. Williams J, Chen Y, Rubin P, et al. The biological basis of a comprehensive grading system for the adverse effects of cancer treatment. Semin Radiat Oncol 2003;13:182-8.

14. Rübe CE, Wilfert F, Uthe D, et al. Modulation of radiation-induced tumour necrosis factor alpha (TNFalpha) expression in the lung tissue by pentoxifylline. Radiother Oncol 2002;64:177-87.

15. Anscher MS, Kong FM, Marks LB, et al. Changes in plasma transforming growth factor beta during radiotherapy and the risk of symptomatic radiation-induced pneumonitis. Int J Radiat Oncol Biol Phys 1997;37:253-8.

16. Anscher MS, Marks LB, Shafman TD, et al. Using plasma transforming growth factor beta-1 during radiotherapy to select patients for dose escalation. J Clin Oncol 2001;19:3758-65.

17. Açil Y, Gierloff M, Behrens C, et al. Effects of zoledronate 
on irradiated bone in vivo: analysis of the collagen types I, V and their cross-links lysylpyridinoline, hydroxylysylpyridinoline and hydroxyproline. Calcif Tissue Int 2013;92:251-60.

18. Atasoy BM, Özgen Z, Bostancı K, et al. The effect of magnesium and vitamin $\mathrm{E}$ pre-treatments on irradiationinduced oxidative injury of cardiac and pulmonary tissues in rats: a randomized experimental study. Anadolu Kardiyol Derg 2012;12:508-14.

19. Tabata C, Kadokawa $Y$, Tabata R, et al. All-trans-retinoic acid prevents radiation- or bleomycin-induced pulmonary fibrosis. Am J Respir Crit Care Med 2006;174:1352-60.

20. Craig VJ, Polverino F, Laucho-Contreras ME, et al. Mononuclear phagocytes and airway epithelial cells: novel sources of matrix metalloproteinase-8 (MMP-8) in patients with idiopathic pulmonary fibrosis. PLoS One 2014;9:e97485.

21. Paun A, Fox J, Balloy V, et al. Combined Tlr2 and Tlr4 deficiency increases radiation-induced pulmonary fibrosis in mice. Int J Radiat Oncol Biol Phys 2010;77:1198-205.

Cite this article as: Yao QW, Wang XY, Li JC, Zhang J. Ophiopogon japonicus inhibits radiation-induced pulmonary inflammation in mice. Ann Transl Med 2019;7(22):622. doi: 10.21037/atm.2019.11.01 